\title{
Integrable field theories from Poisson algebras
}

\author{
Martin Bordemann \\ Fakultät für Physik, Hermann Herder Strasse 3, W-7800 Freiburg, FRG \\ Jens Hoppe and Stefan Theisen \\ Institute for Theoretical Physics, P.O. Box 6380, W-7500 Karlsruhe, FRG
}

Received 24 June 1991

\begin{abstract}
New integrable $1+1$ dimensional classical field theories are found that include infinite dimensional analogues of $N$-particle Toda- and Calogero-Moser systems, as well as non-relativistic theories with an interaction that is polynomial in the first (spatial) derivative of the field. The existence, as well as the involutivity, of an infinite set of independent conserved quantities follows most easily from a $2+1$ dimensional Lax-pair which uses as its underlying infinite dimensional Lie algebra a Poisson algebra of functions in two variables.
\end{abstract}

For the simplest finite dimensional integrable systems, e.g. the Calogero-Moser system with pair potentials proportional to the inverse square distance [1], and the Toda lattice (see e.g. ref. [2] and references therein), with an exponential interaction between nearest neighbours, one not only knows how to write the equations of motion in Lax-form (which implies the existence of sufficiently many independent conserved quantities) but one can also explicitly solve them, by viewing the non-trivial dynamics as a projection [3] of some higher dimensional "free" system.

It seems natural to look for non-trivial infinite dimensional analogues of this desirable situation - an obvious possibility being to simply let the number $N$ (of particles) explicitly go to infinity in the $N$-body hamiltonian (which, in the language of Lie algebra underlying the Lax equation, corresponds to taking the usual [4] $N \rightarrow \infty$ limit of the classical matrix algebras), while more interesting $N \rightarrow \infty$ limits could be related to the Poisson algebra of smooth functions on the sphere [5], or the torus [6-8].

In this letter, we consider a continuous class of field theories whose equation of motion can be written in the form $\mathrm{d} / \mathrm{d} t L=[L, M]$, where $L$ and $M$ are functions of time, and two other variables with respect to which $[L, M]$ is the Poisson bracket $\partial_{1} L \partial_{2} M$ $-\partial_{2} L \partial_{1} M$. In analogy with the above mentioned finite dimensional systems, where the matrix elements of $L$ and $M$ depend (in a very specific way, of course) on a finite number of canonically conjugate coordinates $q_{1}, \ldots, q_{N}, p_{1}, \ldots, p_{N}$, we assume (having refs. [48 ] in mind ) $L$ and $M$ to depend on a $1(+1)$ dimensional field, $q\left(\varphi_{1}, t\right)$, and its canonical conjugate, $p\left(\varphi_{1}\right.$, $t$ ), while depending on $\varphi_{2}$ (and in principle $\varphi_{1}$ as well) explicitly. To be specific, let

$$
\begin{aligned}
& L=p\left(\varphi_{1}, t\right)+e\left(\varphi_{2}\right) F\left(q^{\prime}\left(\varphi_{1}, t\right)\right), \\
& M=m\left[q ; \varphi_{1}\right]-f\left(\varphi_{2}\right) F^{\prime}\left(q^{\prime}\right) .
\end{aligned}
$$

where $e$ and $f$ are phase space independent functions, and ' always means differentiation with respect to the relevant argument (not counting $t$, which we will suppress from now on) Using (1) and (2) it is not difficult to convince oneself that

$\dot{L}=[L, M]:=\frac{\partial L}{\partial \varphi_{1}} \frac{\partial M}{\partial \varphi_{2}}-\frac{\partial L}{\partial \varphi_{2}} \frac{\partial M}{\partial \varphi_{1}}$,

$\cdot=\mathrm{d} / \mathrm{d} t$, provided

$$
\begin{aligned}
& \dot{q}=p, \quad \dot{p}=\kappa q^{\prime \prime} F^{\prime 2}\left(q^{\prime}\right), \\
& e=-f^{\prime}, \\
& F F^{\prime \prime}=\alpha F^{\prime 2},
\end{aligned}
$$


$f^{\prime 2}+(\mu-\alpha f) f^{\prime \prime}=\kappa$,

$\frac{\mathrm{d} m}{\mathrm{~d} \varphi_{1}} F=\mu q^{\prime \prime} F^{\prime 2}$,

and $\alpha, \kappa$, and $\mu$ are real constants.

Hence

$Q_{n}:=\int_{0}^{2 \pi} \frac{\mathrm{d} \varphi_{1}}{2 \pi} \int_{0}^{2 \pi} \frac{\mathrm{d} \varphi_{2}}{2 \pi}(p+e F)^{n}$

is time-independent for any $n \in \mathbb{N}$, provided $e, f, q, p$, and $m$ are periodic functions of their arguments (with period $2 \pi$ ), or fulfil certain (less restricted) boundary conditions, that will be specified below; in fact, one may use compact or non-compact parameterspaces.

As the hamiltonian of an effective $1+1$ dimensional field theory one can e.g. take $H=\frac{1}{2} Q_{2}$, i.e. (assuming $\int_{0}^{2 \pi} e=0$ )

$$
\begin{gathered}
H=\frac{1}{2} \int_{0}^{2 \pi} \frac{\mathrm{d} \varphi}{2 \pi}\left[p^{2}+\lambda F^{2}\left(q^{\prime}\right)\right], \\
\lambda=\int_{0}^{2 \pi} \frac{\mathrm{d} \varphi_{2}}{2 \pi} e^{2}\left(\varphi_{2}\right),
\end{gathered}
$$

with corresponding equations of motion

$\ddot{q}=\kappa q^{\prime \prime} F^{\prime 2}, \quad \kappa=\lambda(\alpha+1)$.

Remarkably, one can show that the $Q_{n}$ Poissoncommute not only with $H$, but also among themselves:

$\left\{Q_{n}, Q_{m}\right\}=\int_{0}^{2 \pi} \frac{\mathrm{d} \varphi}{2 \pi}\left(\frac{\delta Q_{n}}{\delta q(\varphi)} \frac{\delta Q_{m}}{\delta p(\varphi)}-\frac{\delta Q_{n}}{\delta p(\varphi)} \frac{\delta Q_{m}}{\delta q(\varphi)}\right)$

$$
=0, \quad \forall_{m, n} \in \mathbb{N} \text {, }
$$

provided (5)-(7) and certain boundary conditions hold: While (6) can easily be integrated, yielding

$F\left(q^{\prime}\right)=\left(a q^{\prime}+b\right)^{1 /(1-\alpha)}, \quad a, b \in \mathbb{R}$,

for $\alpha=1$, and $a \exp \left(b q^{\prime}\right)$ for $\alpha=1,(7)$, respectively

$g^{\prime 2}=C g^{2 / \alpha}+\kappa, \quad g=f-\mu / \alpha, \quad C \in \mathbb{R}$,

can in general not be integrated in closed form.

However, one can show that (at least) for $0<\alpha \leqslant 1$ (14), with $g^{2 / \alpha}=\left(g^{2}\right)^{1 / \alpha}$, possesses periodic solutions (the period can always be arranged to be $2 \pi$, as $g(p x)$ solves (14), with $c \rightarrow \rho^{2} c$ and $\left.\kappa \rightarrow \rho^{2} \kappa\right)$. If, on the other hand, $g(\varphi)=g_{+}(\varphi-\pi)$, with $g_{+}(-\varphi)$ $=g_{+}(\varphi)$, then $g(0)=g(2 \pi)$, and $\int_{0}^{2 \pi} e=0$; choosing $\mu=\alpha f(0)$ will make $g(0) \quad(=g(2 \pi))=0 \quad$ (and $g^{\prime 2}(0)=\kappa=g^{\prime 2}(2 \pi)$ ); this property (as an alternative to strict periodicity) turns out to be also sufficient for the proof of Poisson-commutativity.

Before sketching our proof of (12), let us give three explicit examples for $H$ (which can be thought of as infinite dimensional analogues of Toda [2], Calogero-Moser $1 / x^{2}[1]$, and in view of its quartic interaction, the Garnier [9] systems ):

$\alpha=1$ :

$F=a \exp \left(b q^{\prime}\right), \quad f=-\sin \varphi_{2}, \quad \mu=0, \quad \kappa=1$,

$L=p+a \cos \varphi_{2} \exp \left(b q^{\prime}\right)$,

$H=\frac{1}{2} \int_{0}^{2 \pi} \frac{\mathrm{d} \varphi}{2 \pi}\left[p^{2}+\frac{1}{2} a^{2} \exp \left(2 b q^{\prime}\right)\right]$,

$\alpha=2$ :

$F=\left(a q^{\prime}+b\right)^{-1}, \quad f\left(\varphi_{2}\right)=\frac{1}{2}\left(\varphi_{2}-\pi\right)^{2}, \quad \mu=\pi^{2}=\kappa$,

$L=p-\left(\varphi_{2}-\pi\right)\left(a q^{\prime}+b\right)^{-1}$,

$H=\frac{1}{2} \int_{0}^{2 \pi} \frac{\mathrm{d} \varphi}{2 \pi}\left(p^{2}+\frac{\pi^{2}}{3} \frac{1}{\left(a q^{\prime}+b\right)^{2}}\right)$,

$\alpha=\frac{1}{2}$

$F=\left(a q^{\prime}+b\right)^{2}$,

$f\left(\varphi_{2}\right)=\sqrt{3} \frac{\pi}{2 K} \operatorname{cn}\left(\frac{2 K}{\pi}\left(\varphi_{2}-\pi\right)\right)$,

$K=K\left(k=\frac{1}{\sqrt{2}}\right)=\frac{1}{4 \sqrt{\pi}}\left[\Gamma\left(\frac{1}{4}\right)\right]^{2}, \quad \mu=0, \quad \kappa=\frac{3}{2}$,

$H=\frac{1}{2} \int_{0}^{2 \pi} \frac{\mathrm{d} \varphi}{2 \pi}\left[p^{2}+\left(a q^{\prime}+b\right)^{4}\right]$.

Also note that choosing $\alpha=1 / N$ leads to

$H=\frac{1}{2} \int_{0}^{2 \pi} \frac{\mathrm{d} \varphi}{2 \pi}\left[p^{2}+\left(a q^{\prime}+b\right)^{N}\right]$.

Quite as $H, Q_{n}$ can also be written as a purely onedimensional object, i.e. without any reference to the $\varphi_{2}$ variable, or the Lax pair, by noting that (7) and 
(5) imply (provided $g g^{\prime m}(0)=g g^{\prime m}(2 \pi)$ for all integer $m$ )

$$
\begin{aligned}
\kappa_{n}:=\int_{0}^{2 \pi} \frac{\mathrm{d} \varphi_{2}}{2 \pi} \exp (n) & =0, \quad n \text { odd }, \\
& =\kappa^{n / 2} I_{n}^{(\alpha)}, \quad n \text { even },
\end{aligned}
$$

where

$I_{2 k}=\frac{(2 k-1)(2 k-3) \ldots(1)}{(2 k-1+\alpha)(2 k-3+\alpha) \ldots(\alpha+1)}$,

So

$$
Q_{n}=\sum_{k=0}^{n}\left(\begin{array}{l}
n \\
k
\end{array}\right) \kappa_{k} \int_{0}^{2 \pi} \frac{\mathrm{d} \varphi}{2 \pi} p^{n-k} F^{k},
$$

and $(10)$ reduces to the identity

$$
\begin{aligned}
& \sum_{k=0}^{[m / 2]}\left(\begin{array}{c}
m \\
2 k
\end{array}\right)\left(\begin{array}{c}
n \\
2 J-2 k
\end{array}\right) I_{2 k} I_{2 J-2 k} \\
& \quad \times\left((2 k-J)(m-2 k)(n+2 k-2 J) \frac{(2 J+\alpha-1)}{(N-2 J-1)}\right. \\
& \quad+(J-k)(m-2 k)(2 J-2 k+\alpha-1) \\
& \quad-k(n+2 k-2 . J)(2 k+\alpha-1)) \\
& \quad=0, \\
& \quad J=1,2, \ldots,\left[\frac{1}{2}(m+n)\right]-1 .
\end{aligned}
$$

The best way to prove (12), however, is to note that $(12)$ is equivalent to

$$
\begin{aligned}
& \int \frac{\mathrm{d} \varphi \mathrm{d} \varphi^{\prime}}{(2 \pi)^{4}}\left\{\exp [\lambda L(\varphi)], \exp \left[\mu L\left(\varphi^{\prime}\right)\right]\right\}=0, \\
& \forall \lambda, \mu \in \mathbb{R} .
\end{aligned}
$$

When calculating the LHS one finds (after several integrations by parts, with respect to $\varphi_{1}$ and/or $\varphi_{1}^{\prime}$ ) that
(5), (6) and (7) are sufficient to obtain 0 ; as part of the calculation one may use that (7) implies

$J_{1}(x)=-\frac{x}{\alpha}\left[J_{2}(x)-\kappa J_{0}(x)\right]$,

if

$J_{k}(x):=\int_{0}^{2 \pi}\left(-g^{\prime}\right)^{k} \exp \left(-x g^{\prime}\right) \frac{\mathrm{d} \varphi_{2}}{2 \pi}$,

with $x$ independent of $\varphi_{2}$, and, as before, either $g(0)=g(2 \pi)=0$, or $g$ and $g^{\prime}$ periodic.

We would like to thank W. Kühn, W. Lang, and M. Schlichenmaier for discussions, and P. Schaller for correspondence. One of us (M.B.) would like to thank the Institute for Theoretical Physics, Karlsruhe, for the kind hospitality.

\section{References}

[1] F. Calogero, Lett. Nuovo Cimento 13 (1975) 411; J. Moser, Adv. Math. 16 (1975) 197.

[2] M. Toda, Theory of nonlinear lattices (Springer, Berlin, $1981)$;

A.M. Perelomov, Integrable systems of classical mechanics and Lie algebras (Birkhäuser, Basel, 1990).

[3] M.A. Olshanetsky and A.M. Perelomov, Lett. Nuovo Cimento 16 (1976) 333; 17 (1976) 97.

[4] V. Kac, Infinite dimensional Lie algebras (Cambridge U.P., Cambridge, 1985).

[5] J. Hoppe, Quantum theory of a massless relativistic surface, MIT Ph.D. Thesis (1982), Elem. Part. Res. J. (Kyoto) 80, No. 3 (1989/90).

[6] D. Fairlie, P. Fletcher and C.N. Zachos, Phys. Lett. B 218 (1989) 203.

[7] J. Hoppe, Intern. J. Mod. Phys. A 4 (1989) 5235.

[8] M. Bordemann, J. Hoppe, P. Schaller and $M$. Schlichenmaier, Commun. Math. Phys. 138 (1991) 209.

[9] R. Garnier, Rend. Circ. Math. Palermo 43 (1919) 155; A. Fordy, S. Wojciechowski and I. Marshall, Phys. Lett. A 113 (1986) 395. 